\title{
A multicentre, cross-sectional study investigating the prevalence of hypertensive disease in patients presenting for elective surgery in the Western Cape Province, South Africa
}

\author{
K van der Spuy, ${ }^{1}$ BSc Hons (Physiotherapy), MB ChB; M Crowther, ${ }^{1}$ MB ChB; M Nejthardt, ${ }^{2}$ BSc Hons (Physiology), MB BCh, DA (SA), \\ FCA (SA); F Roodt, ${ }^{2} \mathrm{MB}$ ChB, FCA (SA); J Davids, ${ }^{3} \mathrm{MB}$ ChB, DA (SA), MMed, FCA (SA); J Roos, ${ }^{4}$ MB ChB, DA (SA), FCA (SA); \\ E Cloete, ${ }^{5} \mathrm{MB}$ ChB, DA (SA), FCA (SA); T Pretorius, ${ }^{6} \mathrm{MB}$ ChB, DA (SA), FCA (SA), MMed; G Davies, ${ }^{6} \mathrm{MB}$ ChB, FCA (SA); \\ J van der Walt, ${ }^{7} \mathrm{MB}$ ChB, DCh (SA), DA (SA), FCA (SA), MMed; C van der Westhuizen, ${ }^{8} \mathrm{MB}$ ChB, DA (SA), FCA (SA), MMed; \\ M Flint, ${ }^{1}$ BSc (Medical Physiology), HSc, MSc, PhD; J Swanevelder, ${ }^{1}$ MB ChB, DA (SA), FCA (SA), MMed, FRCA; \\ B Biccard, ${ }^{1} \mathrm{MB} \mathrm{ChB}, \mathrm{MMedSci}, \mathrm{FCA}(\mathrm{SA}), \mathrm{PhD}$
}

\author{
${ }^{1}$ Department of Anaesthesia and Perioperative Medicine, Groote Schuur Hospital and Faculty of Health Sciences, University of Cape Town, South Africa \\ ${ }^{2}$ Department of Anaesthesia and Perioperative Medicine, Groote Schuur Hospital, Red Cross War Memorial Children's Hospital and Faculty of \\ Health Sciences, University of Cape Town, South Africa \\ ${ }^{3}$ Department of Anaesthesiology, George Regional Hospital, Western Cape, South Africa \\ ${ }^{4}$ Department of Anaesthesiology, Mitchell's Plain Hospital, Cape Town, South Africa \\ ${ }^{5}$ Department of Anaesthesiology, New Somerset Hospital, Cape Town, South Africa \\ ${ }^{6}$ Department of Anaesthesiology, Paarl Provincial Hospital, Western Cape, South Africa \\ ${ }^{7}$ Department of Anaesthesiology, Victoria Hospital, Cape Town, South Africa \\ ${ }^{8}$ Department of Anaesthesiology, Worcester Hospital, Western Cape, South Africa
}

Corresponding author: K van der Spuy (karenvdspuy@gmail.com)

\begin{abstract}
Background. Hypertension is common, affecting over one billion people worldwide. In sub-Saharan Africa, hypertensive disease not only affects the older population but is becoming increasingly prevalent in younger individuals. In South Africa (SA), $>30 \%$ of the adult population has hypertension, making it the single most common cardiovascular risk factor and the predominant contributor to cardiovascular disease and mortality. Elevated blood pressure is the most common perioperative comorbidity encountered in non-cardiac surgical patients, with an overall prevalence of $20-25 \%$, and it remains poorly controlled in low- and middle-income countries. Hypertension in the perioperative setting may adversely affect patient outcome. It therefore not only flags possible perioperative challenges to anaesthesiologists, but also identifies patients at risk of long-term morbidity and mortality.

Objectives. To determine the prevalence and severity of hypertension in elective adult surgical patients in the Western Cape Province, SA. Results. The study population included all elective surgical patients from seven hospitals in the Western Cape during a 1-week period. Hypertension, defined as having had a previous diagnosis of hypertension or meeting the blood pressure criteria of $>140 / 90 \mathrm{mmHg}$, was identified in $51.8 \%$ of patients during preoperative assessment. Significantly, newly diagnosed hypertension was present in $9.9 \%$ of all patients presenting for elective surgery. Although $98.1 \%$ of the known hypertensive patients were on antihypertensive therapy, $36.9 \%$ were inadequately controlled. There are numerous reasons for this, but notably $32.1 \%$ of patients admitted to forgetting to take their medication, making patient factors the most common reason for treatment non-compliance.

Conclusions. The perioperative period may be an important opportunity to identify undiagnosed hypertensive patients. The perioperative encounter may have a significant public health implication in facilitating appropriate referral and treatment of patients with hypertension to decrease long-term cardiovascular complications in SA.
\end{abstract}

S Afr Med J 2018;108(7):590-595. DOI:10.7196/SAMJ.2018.v108i7.13022

Hypertension is common, affecting over one billion people worldwide, and is responsible for over seven million deaths annually. ${ }^{[1]}$ The presence of hypertension increases the risk of myocardial infarction, heart failure, renal failure and cerebrovascular disease. ${ }^{[2]}$ Importantly, in sub-Saharan Africa hypertensive disease not only affects the older population but is becoming increasingly prevalent in younger patient groups. ${ }^{[3]}$ In South Africa (SA), $>30 \%$ of the adult population have hypertension, ${ }^{[4]}$ and it remains the single most common cardiovascular risk factor and the predominant contributor to cardiovascular disease and mortality. ${ }^{[5,6]}$

Elevated blood pressure is the most common perioperative comorbidity encountered in non-cardiac surgical patients, with an overall prevalence of $20-25 \%,{ }^{[7]}$ and it remains poorly controlled in low- and middle-income countries. ${ }^{[8,9]}$ Furthermore, hypertension in the perioperative setting may adversely affect patient outcome. ${ }^{[10]}$ Hypertension therefore poses perioperative challenges to anaesthesiologists, while simultaneously identifying patients at risk of long-term morbidity and mortality. ${ }^{[1,12]}$

Hypertension is most frequently diagnosed and treatment initiated in the primary healthcare setting. However, in a resourcelimited environment, the perioperative period gives clinicians a unique opportunity to identify patients with hypertensive disease, educate patients about the disease, and initiate appropriate therapy. Furthermore, this period provides the opportunity to refer patients for further investigation or follow-up at peripheral healthcare centres for ongoing management, thus aiding the decentralisation 
of chronic, long-term care. The efficient identification and diagnosis of hypertension in the perioperative period could therefore be seen as an effective utilisation of planned surgical admission by simultaneously addressing a primary healthcare need, and this may serve as an efficient healthcare strategy in reducing long-term cardiovascular morbidity and mortality. ${ }^{[13,14]}$ Identification and/ or optimisation of hypertensive management in the perioperative period is an attractive healthcare intervention in a resource-limited environment such as SA, particularly when considering difficulties with primary healthcare access and treatment compliance.

\section{Objectives}

The primary objective of this study was to describe the prevalence and severity of hypertension in adult patients presenting for non-cardiac, non-obstetric elective surgery in all surgical disciplines at seven hospitals in the Western Cape Province, SA, in order to determine whether perioperative screening can be used to supplement primary healthcare management of hypertension through developing effective strategies for the diagnosis and management of hypertension in patients presenting for elective surgery. The secondary objectives were to identify hypertension-associated target organ damage and risk factors associated with hypertension, and to assess compliance with prescribed hypertensive therapy.

\section{Methods}

This was a multicentre, prospective, observational study conducted at seven hospitals in the Western Cape: one tertiary institution, Groote Schuur Hospital, and six secondary institutions, George, Mitchell's Plain, New Somerset, Paarl, Victoria and Worcester hospitals. Ethics approval was obtained for all institutions (ref. nos HREC 661/2016 and 708/2016 and NHRD WC_2016RP55_876), and written informed consent was obtained prior to patient enrolment in the study. The trial was registered on the South African National Clinical Trial Register (NCT03157661).

All adult, non-cardiac, non-obstetric patients admitted the day before elective surgery during the study period were eligible for inclusion. Exclusion criteria were patient refusal, day-case surgery (as no preceding day preoperative assessment was possible) and patients not requiring an anaesthetic. Recruitment was from $07 \mathrm{~h} 00$ on Monday to $19 \mathrm{~h} 00$ on Friday of the week chosen for the study.

Data were collected by anaesthesia medical officers, registrars and consultants assigned to each of the surgical lists at the various institutions. Routine preoperative information was recorded on a specifically designed paper case report form and then captured onto the Research Electronic Data Capture (REDCap) web-based application. Compliance with medical therapy was assessed using the Morisky Medication Adherence Questionnaire. ${ }^{[15,16]}$ A positive response to two or more of the four questions was considered to indicate non-compliance with antihypertensive treatment.

Assessment of preoperative hypertension was evaluated using the South African Hypertension Practice Guideline. ${ }^{[2]}$ All blood pressure measurements were performed with an appropriately sized non-invasive blood pressure cuff, using an automated oscillometric method of blood pressure measurement. If the patient was found to have a systolic blood pressure of $\geq 140 \mathrm{mmHg}$ or a diastolic blood pressure of $\geq 90 \mathrm{mmHg}$, two further measurements were performed at least 5 minutes apart. The lowest of the three readings was taken as the preoperative blood pressure. Patients who still had a systolic blood pressure of $\geq 140 \mathrm{mmHg}$ or a diastolic blood pressure of $\geq 90 \mathrm{mmHg}$ after these three blood pressure readings were considered to be hypertensive. ${ }^{[4]}$
Categorical variables were described as proportions and compared using $\chi^{2}$ tests and Fisher's exact tests, as appropriate. Continuous variables were described as means and standard deviations (SDs) or medians and interquartile ranges (IQRs) and compared using $t$-tests or one-way analysis of variance, as appropriate. Data were analysed using the Statistical Package for the Social Sciences (SPSS) version 24 (SPSS, USA).

\section{Results}

The seven participating hospitals and numbers of patients screened and recruited with complete data are shown in Table 1. Patient recruitment and the prevalence and control of hypertension are shown in Fig. 1. Of the 397 patients who were screened, 5 refused to participate and 4 did not meet the inclusion criteria. Six patients were excluded from the analysis owing to incomplete datasets. Analysis was possible on full datasets of $382 / 388$ consenting patients (98.5\%).

Of the patients, 160 (41.9\%) had previously diagnosed hypertension, while newly diagnosed hypertension was present in a further 38 (9.9\%). The prevalence of hypertension, defined as having a previous diagnosis of hypertension or meeting the blood pressure criteria of $>140 / 90 \mathrm{mmHg}$, was therefore $198 / 382$ (51.8\%, 95\% confidence interval (CI) 46.8 - 56.8) in the preoperative assessment.

The characteristics of the recruited patients are shown in Table 2. Hypertensive patients were older, carried more risk factors for

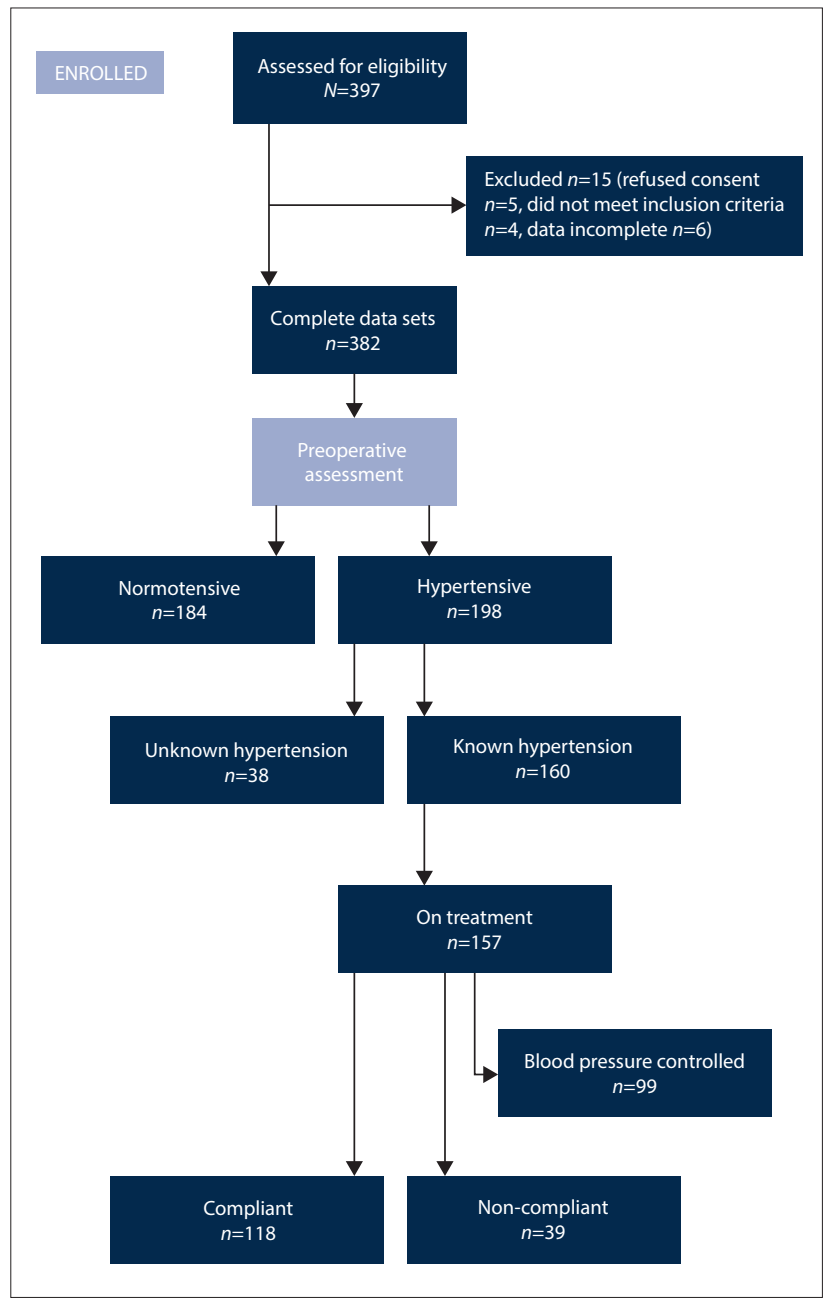

Fig. 1. PRISMA diagram depicting the study recruitment process. 


\section{Table 1. Participating hospitals}

\begin{tabular}{llll}
\hline Institution & Level of care & $\begin{array}{l}\text { Patients screened } \\
(\boldsymbol{N}=\mathbf{3 9 7}), \boldsymbol{n}\end{array}$ & $\begin{array}{l}\text { Patients recruited with } \\
\text { complete data }(\boldsymbol{N = 3 8 2}), \boldsymbol{n}\end{array}$ \\
\hline George Provincial Hospital & Secondary & 49 & 49 \\
Groote Schuur Hospital & Tertiary & 187 & 179 \\
Mitchell's Plain Hospital & Secondary & 28 & 27 \\
New Somerset Hospital & Secondary & 45 & 42 \\
Paarl Hospital & Secondary & 46 & 46 \\
Victoria Hospital & Secondary & 12 & 10 \\
Worcester Hospital & Secondary & 30 & 29
\end{tabular}

Table 2. Characteristics of the study population

\begin{tabular}{|c|c|c|c|c|}
\hline & Patients $(N=382)$ & Normotensive $(N=184)$ & Hypertensive $(N=198)$ & $p$-value \\
\hline Age (years), mean (SD) & $50(16.1)$ & $41.2(14.3)$ & $58.5(12.9)$ & $<0.001$ \\
\hline Gender male, $n(\%)$ & $146(38.2)$ & $76(41.3)$ & $70(35.4)$ & 0.248 \\
\hline ASA status ( $N=379), n(\%)$ & & & & $<0.001$ \\
\hline I & $127(33.5)$ & $107(59.1)$ & $20(10.1)$ & \\
\hline II & $187(49.3)$ & $62(34.3)$ & $125(63.1)$ & \\
\hline III & $59(15.6)$ & $11(6.1)$ & $48(24.2)$ & \\
\hline IV & $6(1.6)$ & $1(0.6)$ & $5(2.5)$ & \\
\hline \multicolumn{5}{|c|}{ Risk factors for hypertension, $n$ (\%) } \\
\hline BMI $(N=358)$ & $28.2(7.2)$ & $26.6(6.8)$ & $29.7(7.3)$ & $<0.001$ \\
\hline Smoking & $160(41.9)$ & $89(48.4)$ & $71(35.9)$ & 0.778 \\
\hline Dyslipidaemia & $48(12.6)$ & $7(3.8)$ & $41(20.7)$ & $<0.001$ \\
\hline NIDDM & $19(5.0)$ & $5(2.7)$ & $14(7.1)$ & 0.060 \\
\hline IDDM & $42(11.0)$ & $6(3.3)$ & $36(18.2)$ & $<0.001$ \\
\hline Men $>55$ years & $55(14.4)$ & $18(9.8)$ & $37(18.7)$ & 0.014 \\
\hline Women $>65$ years & $46(12.0)$ & $7(3.8)$ & $39(19.7)$ & $<0.001$ \\
\hline \multicolumn{5}{|l|}{ Target organ damage, $n(\%)$} \\
\hline Left ventricular hypertrophy & $39(10.2)$ & $4(2.2)$ & $35(17.7)$ & $<0.001$ \\
\hline Coronary artery disease & $14(3.7)$ & $2(1.1)$ & $12(6.1)$ & 0.012 \\
\hline Heart failure & $5(1.3)$ & $1(0.5)$ & $4(2.0)$ & 0.204 \\
\hline Chronic kidney disease & $27(10.4)$ & $2(2.0)$ & $25(15.7)$ & $<0.001$ \\
\hline CVA/TIA & $6(1.6)$ & $1(0.5)$ & $5(2.5)$ & 0.120 \\
\hline Peripheral arterial disease & $8(2.1)$ & $2(1.1)$ & $6(3.0)$ & 0.185 \\
\hline Retinopathy & $8(2.1)$ & $0(0.0)$ & $8(4.0)$ & 0.008 \\
\hline \multicolumn{5}{|l|}{ Other comorbid disease } \\
\hline COPD/asthma & $36(9.4)$ & $13(7.1)$ & $23(11.6)$ & 0.161 \\
\hline HIV/AIDS & $24(6.3)$ & $16(8.7)$ & $8(4.0)$ & 0.090 \\
\hline
\end{tabular}

Table 3. Classification of the severity of hypertension* $(N=198)$

\begin{tabular}{ll} 
Table 3. Classification of the severity of hypertension* $(\boldsymbol{N = 1 9 8 )}$ & Hypertensive, $\boldsymbol{n}(\mathbf{\%})$ \\
\hline & $99(50.0)$ \\
\hline Normotensive & $66(33.3)$ \\
Grade 1: mild (SBP $140-159$ or DBP $90-99 \mathrm{mmHg})$ & $21(10.6)$ \\
Grade 2: moderate (SBP $160-179 \mathrm{mmHg}$ or DBP $100-109 \mathrm{mmHg})$ & $12(6.1)$ \\
Grade 3: severe (SBP $\geq 180 \mathrm{mmHg}$ or DBP $\geq 110 \mathrm{mmHg})$ & \\
SBP $=$ systolic blood pressure; DBP $=$ diastolic blood pressure. & ${ }^{*}$ Classification of hypertension as per the 2014 South African Hypertension Practice Guideline. ${ }^{[2]}$
\end{tabular}

hypertension and had more comorbidities as reflected by the higher American Society of Anesthesiologists grading. Hypertensive patients had significantly more target organ damage, specifically coronary artery disease, heart failure, advanced retinopathy, cerebrovascular disease, chronic renal disease, peripheral arterial disease, diabetes and dyslipidaemia.
The severity of hypertension is shown in Table 3. Of all the hypertensive patients, 99/198 (50.0\%, 95\% CI 43.0 - 57.0) were found to have a blood pressure $>140 / 90 \mathrm{mmHg}$. Despite the vast majority of known hypertensive patients (157/160, 98.1\%) being on antihypertensive therapy preoperatively, blood pressure control was inadequate in $61 / 160(38.1 \%, 95 \%$ CI 31.2 - 46.3). 
Table 4 lists the most common antihypertensive therapies in the study population. Of the patients who presented for surgery with a diagnosis of hypertension, 39/157 reported treatment noncompliance $(24.8 \%, 95 \%$ CI 18.2 - 31.8) (Table 5). A third of patients (50/156) taking antihypertensive medication admitted to forgetting to take their medication. Patient factors were the most common cause of treatment non-compliance.

\section{Discussion \\ Principal findings}

Five out of every ten patients presenting for elective surgery in the Western Cape are hypertensive. Of these, 20\% are undiagnosed and $40 \%$ are inadequately controlled. This study suggests that the perioperative period may be an important opportunity to identify undiagnosed hypertension as well as improve the management of known hypertensive patients in SA.

Once a patient is diagnosed with hypertension, access to medication in the community is good, but patient compliance with therapy becomes the more important determinant of subsequent hypertensive control.

These data suggest that the perioperative period could supplement primary healthcare services, through perioperative screening, treatment initiation and referral. This needs to be coupled with an appropriate educational programme to ensure subsequent patient compliance with therapy on discharge. This dual-pronged approach to hypertension in surgical patients has the potential for a large public health benefit in SA.

\section{Implications of the study}

It is estimated that 3.5 billion adults, or $60 \%$ of the world's population, is hypertensive. ${ }^{[17]}$ In the African region, the prevalence of hypertension

\begin{tabular}{ll}
\multicolumn{3}{l}{ Table 4. Most common antihypertensive therapies used } \\
\hline Antihypertensive treatment & $\boldsymbol{n}(\%)$ \\
\hline Diuretic & $124 / 160(77.5)$ \\
ACE-I/ARB & $97 / 160(60.6)$ \\
Beta blocker & $61 / 160(38.1)$ \\
Calcium channel blocker & $46 / 160(28.8)$ \\
Alpha blocker & $6 / 160(3.8)$ \\
Other & $4 / 160(2.5)$ \\
ACE-I = angiotensin-converting enzyme inhibitor; ARB = angiotensin receptor blocker.
\end{tabular}

is estimated at $46 \%$ for adults aged $\geq 25$ years. The number of adults with hypertension is predicted to increase by $\sim 60 \%$ by 2025 , with a disproportionately high prevalence in developing countries. ${ }^{[18]}$

In sub-Saharan Africa, despite the high burden imposed by communicable diseases, hypertension has emerged as a significant medical and public health problem and is regarded as one of the continent's greatest health challenges after HIV/AIDS. ${ }^{[1]}$ It is estimated that if the 10 - 20 million people who are believed to have hypertension in sub-Saharan Africa were treated effectively, about 250000 deaths could be prevented annually. ${ }^{[19]}$

According to the South African Hypertension Practice Guideline, ${ }^{[2]}$ $30.4 \%$ of the SA adult population has hypertension. This chronic disease is regarded as the single most prevalent cardiovascular risk factor and as a predominant contributor to cardiovascular diseaserelated morbidity and mortality. ${ }^{[1]}$ In the 2015 SA mortality statistics, cerebrovascular disease ranked third, accounting for $5.0 \%$ of national natural causes of death, heart disease ranked fifth, accounting for $4.8 \%$, and hypertension-related diseases ranked seventh, accounting for $4.2 \%{ }^{[20]}$

Although hypertension is not directly linked to poor perioperative outcomes, it is associated with long-term cardiovascular morbidity and mortality. ${ }^{[2]}$ Treating hypertension improves long-term outcome. ${ }^{[13]}$ This study suggests that perioperative evaluation of blood pressure has the potential to: (i) newly diagnose hypertension in $10 \%$ of all adult patients presenting for elective surgery; (ii) provide surveillance for the adequacy of management of hypertension in the community; and (iii) play an active role in the management of hypertension using predefined interventions ${ }^{[21]}$ to improve both understanding and control of hypertension in as many as $50 \%$ of patients who present for all elective surgery.

The global volume of surgery, based on population statistics from 2005 to 2013, estimates an average imputed surgical rate of 5227 per 100000 population. ${ }^{[22]}$ With a conservative estimate of 20000 elective, adult non-cardiac, non-obstetric surgical procedures per annum in the Western Cape (B M Biccard, unpublished data, June 2018), as many as $2000(10 \%)$ new cases of hypertension could be diagnosed perioperatively. Furthermore, a total of $8000(40 \%)$ of these patients would require further therapy optimisation in the perioperative period. The population attributable risk associated with hypertension for stroke in South Africa is $~ 50 \%$, while that for ischaemic heart disease is $40 \% \cdot{ }^{[21]}$ Optimisation of hypertension could therefore prevent 125 strokes and 244 coronary events in this

Table 5. Incidence of and reasons for non-compliance with hypertensive therapy ${ }^{\star}$

\begin{tabular}{|c|c|c|}
\hline & $\begin{array}{l}\text { Hypertensive pati } \\
\text { treatment, } n / \text { total }\end{array}$ & $\% ; 95 \%$ CI \\
\hline Compliant & $117 / 156$ & $75.0 ; 68.2-81.2$ \\
\hline Non-compliant & $39 / 156$ & $25.0 ; 18.2-31.8$ \\
\hline \multicolumn{3}{|l|}{ Standardised questions to elicit non-compliance } \\
\hline Do you ever forget to take your medicine? & $50 / 156$ & $32.1 ; 24.7-39.3$ \\
\hline Are you careless at times about taking your medicine? & $35 / 154$ & $22.7 ; 16.1-29.3$ \\
\hline When you feel better, do you sometimes stop taking your medicine? & $20 / 155$ & $12.9 ; 7.6-18.2$ \\
\hline Sometimes if you feel worse when taking your medication, do you stop taking it? & $20 / 155$ & $12.9 ; 7.6-18.2$ \\
\hline \multicolumn{3}{|l|}{ Reasons for non-compliance $(N=39)$} \\
\hline Health system & $3 / 39$ & $7.9 ; 0.0-16.5$ \\
\hline Condition & $8 / 39$ & $21.1 ; 8.1-34.0$ \\
\hline Patient & $22 / 39$ & $57.9 ; 42.2-73.4$ \\
\hline Therapy & $6 / 39$ & $15.8 ; 4.2-27.3$ \\
\hline Socioeconomic & $5 / 39$ & $13.2 ; 2.4-23.9$ \\
\hline
\end{tabular}


population annually, based on the prevalence of stroke and coronary heart disease in our population (2.5\% and $6.1 \%$, respectively).

\section{Implications for SA}

When considering national statistics, with an average imputed surgical rate of 5227 per 100000 population ${ }^{[23]}$ and estimating that $50 \%$ of all surgeries are for adults aged $\geq 18$ years of age and older (2 614 per 100000 population), as many as $261(10 \%)$ per 100000 new cases of hypertension could be diagnosed perioperatively annually, and the opportunity will exist to optimise hypertensive treatment in up to $1046(40 \%)$ per 100000 patients annually. This approach to perioperative hypertension therefore has the potential to prevent 16 strokes and 32 coronary events per 100000 of the adult population in SA, annually.

\section{Study strengths and limitations}

This was a multicentre, prospective observational study of hypertension in the Western Cape. The fact that we were able to follow the SA hypertension guidelines in confirming the diagnosis of hypertension on the day prior to surgery in this study increases our confidence that our results reflect the true burden of hypertensive disease in preoperative surgical patients. We expect the data to be broadly generalisable across the Western Cape, and possibly across SA for patients from similar social circumstances.

It is possible that the prevalence of hypertension may be overestimated in this cohort. Although the evaluation of hypertension was made in elective surgical patients on the day preceding surgery, it is possible that some of the patients may have been anxious, and hence spuriously fulfilled the diagnostic criteria. Furthermore, this study excluded all emergency cases, so it was not possible to obtain a true prevalence of hypertension in patients presenting for all surgery. However, we would expect the prevalence of hypertension to be higher in patients presenting for emergency surgery, as they are more likely than the elective population to have comorbid disease.

Finally, the information related to compliance with medical therapy should be viewed with some caution, as it is based on a relatively small sample size.

\section{Conclusions}

We believe that in SA there is a significant potential for public health interventions in the perioperative period. In particular, we have demonstrated a unique diagnostic and therapeutic opportunity in patients with hypertension. Further research is needed into other comorbidities such as anaemia and diabetes, where similar potential benefits may apply.

Acknowledgements. We wish to acknowledge and thank all the anaesthetic registrars and consultants involved in the study design, planning and organisation, as well as all who were involved in data collection and capture at the various institutions included in our study: George Hospital, Groote Schuur Hospital, Mitchell's Plain Hospital, New Somerset Hospital, Paarl Hospital, Victoria Hospital and Worcester Hospital. We also thank the Department of Anaesthesia and Perioperative Medicine at Groote Schuur Hospital for accommodating the roster and other logistic changes, making this study possible.

Author contributions. This submission has 14 authors from a multicentre, prospective, observational study of seven hospitals in the Western Cape, SA. We have itemised their contributions according to the International Committee of Medical Journal Editors criteria. KvdS: overall conception and design of the Hypertension and Surgery (HaS) study, acquisition of data at Groote Schuur Hospital, interpretation, drafting and critical revising of the work, final approval of the version to be published, agree to be accountable for all aspects, accuracy and integrity of the work; MC: overall conception and design of the $\mathrm{HaS}$ study, acquisition of data at Groote Schuur Hospital, interpretation, critical revising of the work, final approval of the version to be published, agree to be accountable for all aspects, accuracy and integrity of the work; MN: overall conception and design of the HaS study, analysis and interpretation, drafting and critical revising of the work, final approval of the version to be published, agree to be accountable for all aspects, accuracy and integrity of the work; FR: overall conception and design of the HaS study, acquisition of data at Mitchell's Plain Hospital, analysis and interpretation, critical revising of the work, agree to be accountable for all aspects, accuracy and integrity of the work; JD: acquisition of data at George Hospital, critical revising of the work, final approval of the version to be published, agree to be accountable for all aspects, accuracy and integrity of the work: JR: acquisition of data at Mitchell's Plain Hospital, critical revising of the work, final approval of the version to be published, agree to be accountable for all aspects, accuracy and integrity of the work; EC: acquisition of data at New Somerset Hospital, critical revising of the work, final approval of the version to be published, agree to be accountable for all aspects, accuracy and integrity of the work; TP: acquisition of data at Paarl Provincial Hospital, critical revising of the work, final approval of the version to be published, agree to be accountable for all aspects, accuracy and integrity of the work; GD: acquisition of data at Paarl Provincial Hospital, critical revising of the work, final approval of the version to be published, agree to be accountable for all aspects, accuracy and integrity of the work; JvdW: acquisition of data at Victoria Hospital, critical revising of the work, final approval of the version to be published, agree to be accountable for all aspects, accuracy and integrity of the work; CvdW: acquisition of data at Worcester Hospital, critical revising of the work, final approval of the version to be published, agree to be accountable for all aspects, accuracy and integrity of the work; MF: acquisition of data at Groote Schuur Hospital, critical revising of the work, final approval of the version to be published, agree to be accountable for all aspects, accuracy and integrity of the work; JS: overall conception and design of the HaS study, critical revising of the work, final approval of the version to be published, agree to be accountable for all aspects, accuracy and integrity of the work; BB: overall conception and design of the Has study, analysis of results, drafting and critical revising of the work, final approval of the version to be published, agree to be accountable for all aspects, accuracy and integrity of the work.

Funding. This study was supported by departmental resources.

Conflicts of interest. None.

1. Norman R, Gaziano T, Laubscher R, Steyn K, Bradshaw D. Estimating the burden of diseas attributable to high blood pressure in South Africa in 2000. S Afr Med J 2007;97(8 Pt 2):692-698. 2. Seedat YK, Rayner BL, Veriava Y. South African Hypertension Practice Guideline 2014. Cardiovasc Afr 2014;25(6):288-294. https://doi.org/10.5830\%2Fcvja-2014-062

3radshaw D, Norman R, Lewin S, et al. Strengthening public health in South Africa: Building a stronger evidence base for improving the health of the nation. S Afr Med J 2007;97(8):643-651.

4. Mayosi BM, Flisher AJ, Lalloo UG, Sitas F, Tollman SM, Bradshaw D. The burden of noncommunicable diseases in South Africa. Lancet 2009;374(9693):934-947. https://doi.org/10.1016\%2 Fs $0140-6736 \% 2809 \% 2961087-4$

5. Seedat Y. Hypertension in developing nations in sub-Saharan Africa. J Hum Hypertens 2000;14(1011):739-747. https://doi.org/10.1038\%2Fsj.jhh.1001059

6ennison CR, Peer N, Steyn K, Levitt NS, Hill MN. Determinants of hypertension care and control 6. Dennison CR, Peer N, Steyn K, Levitt NS, Hill MN. Determinants of hypertension care
among peri-urban Black South Africans: The HiHi study. Ethn Dis 2007;17(3):484-491.

7. Manolis A, Erdine S, Borghi C, Tsioufis K. ESH Clinical Newsletter: Perioperative screening and management of hypertensive patients. J Hypertens 2011;15(3):28-32. http://www.eshonline.org/eshcontent/uploads/2015/03/ESH-Clinical-Practice-Newsletters_2011.pdf (accessed 9 June 2018).

8. Opie LH, Seedat YK. Hypertension in sub-Saharan African populations. Circulation 2005;112(23):3562-3568. https://doi.org/10.1161\%2Fcirculationha.105.539569

9. Lloyd-Sherlock P, Beard J, Minicuci N, Ebrahim S, Chatterji S. Hypertension among older adults in low- and middle-income countries: Prevalence, awareness and control. Int J Epidemiol 2014;43(1):116128. https://doi.org/10.1093\%2Fije\%2Fdyt215 
10. James MF, Dyer RA, Rayner B. A modern look at hypertension and anaesthesia. J Endocrinol Metab Diabetes S Afr 2014;19(2):56-61. https://doi.org/10.1080\%2F22201173.2011.10872770

11. Sear JW. Perioperative control of hypertension: When will it adversely affect perioperative outcome? Cear JW. Perioperative control of hypertension: When will it adversely affect perioperative
Curr Hypertens Rep 2008;10(6):480-487. https://doi.org/10.1007\%2Fs11906-008-0090-2 12. Hartle A, McCormack T, Carlisle J, et al. The measurement of adult blood pressure and management of
hypertension before elective surgery: Joint Guidelines from the Association of Anaesthetists of Great hypertension before elective surgery: Joint Guidelines from the Association of Anaesthetists of Great
Britain and Ireland and the British Hypertension Society. Anaesthesia 2016;71(3):326-337. https://doi Britain and Ireland and the
org $/ 10.1111 \% 2$ Fanae. 13348

13. Mittal BV, Singh AK. Hypertension in the developing world: Challenges and opportunities. Am J Kidney Dis 2010;55(3):590-598. https://doi.org/10.1053\%2Fj.ajkd.2009.06.04

4. Coovadia H, Jewkes R, Barron P, Sanders D, McIntyre D. The health and health system of South Africa: Historical roots of current public health challenges. Lancet 2009;374(9692):817-834. https://doi. org/10.1016\%2Fs0140-6736\%2809\%2960951-x

15. Morisky DE, Green LW, Levine DM. Concurrent and predictive validity of a self-reported measure of medication adherence. Med Care 1986;24(1):67-74. https://doi.org/10.1097\% 2F00005650-198601000-00007

16. Krousel-Wood M, Thomas S, Muntner P, Morisky D. Medication adherence: A key factor in achieving blood pressure control and good clinical outcomes in hypertensive patients. Curr Opin Cardio 2004;19(4):357-362. https://doi.org/10.1097\%2F01.hco.0000126978.03828.9e

17. Forouzanfar MH, Liu P, Roth GA, et al. Global burden of hypertension and systolic blood pressure of at least 110 to $115 \mathrm{mmHg}$ 1990 2015. JAMA 2017:317(2):165-182. https:/doioro/10.1001/ jama.2016.19043
18. Cappucino FP, Miller MA. Cardiovascular disease and hypertension in Sub-Saharan Africa: Burden, risk and interventions. Intern Emerg Med 2016;11(3):299-305. https://doi.org/10.1007\%2Fs11739-016-

19. Cooper RS, Rotimi C, Kaufman JS, et al. Hypertension treatment and control in sub-Saharan Africa: The epidemiological basis for policy. BMJ 1998;316(7131):614-617. https://www.ncbi.nlm.nih.gov/ mc/articles/PMC1112640

20. OECD. Basic Statistics of South Africa, 2011. In: OECD Economic Surveys: South Africa 2013. OECD Publishing. https://doi.org/10.1787/eco_surveys-zaf-2013-en

21. Peer N. Hypertension: Now is the time for action. Chronic Diseases of Lifestyle Unit at the Medical Research Council 2013: https://www.phasa.org.za/hypertension-now-is-the-time-for-action/ (accessed 9 June 2018).

22. Weiser TG, Haynes AB, Molina G, Lipsitz SR, et al. Estimate of the global volume of surgery in 2012: An assessment supporting improved health outcomes. Lancet 2015;385(Suppl 2):S11. https://doi.org/1 0.1016\%2Fs0140-6736\%2815\%2960806-6

23. Biccard BM, Madiba TE. The South African Surgical Outcomes Study: A 7-day prospective observational cohort study. S Afr Med J 2015;105(6):465-475. https://doi.org/10.7196/SAMJ.9435

Accepted 9 January 2018. 